\section{Authorship and misconduct}

SIR — The leading article "What to do about scientific misconduct"1 arrived in Canada at the same time as headlines in a major national newspaper announced the results of an inquiry into a professor's shooting rampage at Montreal's Concordia University ${ }^{2}$. The inquiry concluded that there was some truth to his claim that his senior colleagues had engaged in "misappropriation of authorial credit", and pointed to the "risk that a productiondriven research culture will tempt people to engage not only in undesirable modalities of research, but also in actual falsification and fraud". This view and your remedy, that there should be a "weakening of links between personal success and publication", do not really get to the heart of the problem, and, if misconstrued, might even make the problem worse.

There is nothing wrong with a link between personal success and publication. The problem is that quality evaluation in research is highly error-prone. Any system that is error-prone has to be designed taking error-proneness into account. Conventional peer-review does not do this. An alternative, bicameral review, has been proposed ${ }^{3-5}$. The golden rules for working in error-prone environments are (1) use the most objective parameters, and (2) hedge your bets. Under bicameral review, research grant applications are divided into two parts, prospective and retrospective. The prospective part (work proposed) is short and is evaluated by the granting agency, solely on the basis of budget justification. The retrospective part (track record) is evaluated by peers who rate it on the basis of productivity per research dollar received. Finally, rather than a sharp cut-off point below which no funds are awarded, a sliding scale is used.

One cannot get rid of competition. But, if implemented, bicameral review might, over the years, decrease the competitive pressure on researchers and enhance communication and collaboration. Civility would return to the research enterprise.

Donald R. Forsdyke

Department of Biochemistry,

Queen's University,

Kingston, Ontario,

CanadaK $7 L$ 3N6

SIR - As the importance given to publications is central to fraud and other forms of scientific misconduct, the suggestion ${ }^{1}$ that the link between papers and personal success should be weakened is a logical step. Indeed, career advancement for scientists depends heavily on the number of publications regardless of the first author/ co-author ratio. Restoring the original meaning to the word 'author' would encourage scientists to concentrate on their own work rather than to search for convenient collaborations.

Tenure committees and granting agencies should concentrate on first or single author papers, and reviewers of manuscripts and research projects should promote responsible authorship. An individual's name becomes known by appearing in that first position and not among the so-called co-authors. This suggestion conforms to previous proposals for fixing an annual limit to papers per scientist or evaluating the career of an individual through a few articles. The cynical and seemingly robust objection that junior scientists would never appear as first authors can be overcome provided they receive appropriate advice and protection from scientific institutions and research councils.

Despite a possible increase in the disputes about who should go first, a proper use of the word author would lead to a more realistic judgement of personal creativity, an attenuation of the prevalent curricular inflation and article devaluation and an acknowledgement of the secondary role of co-authors. This in turn will result in shorter authors' lists with a positive impact on the scientific literature (less space used, more ease). That coauthorship is indeed secondary is illustrated by its widespread use as a token to pay for any contribution/favour or to flatter a superior.

In line with the earlier pungent comments by DeFelice ${ }^{6}$, it can be said that the scientific establishment has resisted criticism of its behaviour patterns and publication trends because most scientists automatically close ranks to protect themselves. It is clear, however, that the publication game rules need to be reappraised in order to rescue the honour of the scientific profession - entrepreneurial science notwithstanding.

\section{Horacio Rivera}

Division de Genetica,

Instituto Mexicano del Seguro Social, Ap. Postal 1-3838,

Guadalajara,

Mexico

SIR - You ask how we can weaken the link between a researcher's success and his or her list of publications ${ }^{7}$. One way to do so would be reopen the debate about the connection between spurious coauthorship and scientific fraud ${ }^{6}$. The pressure to produce a tidy result is enormous. Thus, when a laboratory chief requires data for grants or for lectures, the temptation to edit naturally arises. The best results for these purposes are those that fit in with other data or those that anticipate a major advance. The last thing a subordinate wants to find or a laboratory chief wants to hear is something offbeat or discouraging. If laboratory chiefs become co-authors of data they are not familiar with, which is too often the case, fraud can result. Data editing, indirectly encouraged, goes undetected. Should the laboratory chief invite a colleague on the fringe of the work to become an author, the potential for fraud increases. This common practice introduces another voice for what the data should be rather than what they are, another vote for consensus (or discovery) even if there is none.

If you doubt that this happens, invite comments from graduate students, postdocs and senior associates from big laboratories. Spurious co-authorship is a major cause of scientific misconduct, and even its innocent or well-meaning forms are harmful to the community.

Louis J. DeFelice

Department of Anatomy and Cell Biology,

Emory University School of Medicine,

Atlanta, Georgia 30322-3030, USA

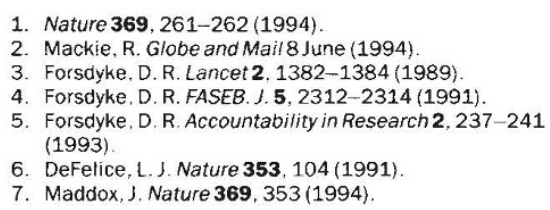

\section{Not so humane}

SIR - We would like to bring to your attention a patent violation of animal rights, paradoxically caused by extreme human care.

The episode took place last January in an Italian medical institute, where a precious primate was kept in temporary custody. The specimen was an adult female, in the latest stage of pregnancy. Because of the considerable value of both mother and fetus, labour was monitored throughout by the use of a cardiotocograph. For this purpose, the mother was restrained in an uncomfortable supine position. For greater safety, uterine dilation was monitored by manual inspection, about 6 times an hour, each time causing evident pain to the animal. Finally, just before delivery, episiotomy was performed to reduce the risks of delivery.

Both mother and baby are now perfectly well, but we believe that this and similar cases should be brought to the attention of whoever cares for animal welfare.

We certify that what is reported is true: one of us is the primate in question.

Lucia Galli-Resta

Istituto di Neurofisiologia,

Giovanni Resta

Istituto di Elaborazione dell'Informazione, Consiglio Nazionale delle Ricerche,

Pisa,

Italy 